\title{
Fast phase reconstruction in off-axis digital holography with zero-order term suppression
}

\author{
GuAngyu LUAN ${ }^{1 *}$, MingGuang SHAN ${ }^{2 *}$, Zhi ZHONG ${ }^{2}$, Bai XIA ${ }^{2}$ \\ ${ }^{1}$ College of Electrical and Information, Heilongjiang Bayi Agricultural University, \\ Daqing, Heilongjiang, 163319, China \\ ${ }^{2}$ College of Information and Communication Engineering, Harbin Engineering University, \\ Harbin, Heilongjiang, 150001, China
}

*Corresponding authors: smgsir@gmail.com, luanguangyu@126.com

\begin{abstract}
A fast phase reconstruction method (FPRM) employing a free-sample hologram can improve the efficiency of phase reconstruction in off-axis digital holography. However, the space-bandwidth product is still confined by spectrum aliasing in the hologram owing to the zero-order term. In this paper, we propose an FPRM that features an efficient zero-order term suppression method called the average gray that can eliminate spectrum shifting. We can implement phase reconstruction by considering both speed and the space-bandwidth product. We verified the validity of our approach for off-axis digital holography using laser and white-light illumination.
\end{abstract}

Keywords: digital holography, phase retrieval, off-axis, zero-order suppression, filtering.

\section{Introduction}

Off-axis digital holography (DH) has garnered a great deal of interest from the scientific community over the past decade because it can provide a quantitative phase imaging in one shot, thus allowing for acquisition at the camera's frame rate [ $\underline{1}-\underline{5}]$. In off-axis DH, the Fourier transform method is usually employed to implement phase reconstruction because the zero-order and conjugate-order $(+1$ st and -1 st diffraction orders) terms generated by the recorded hologram can be separated in the spectral domain. However, the computational process, especially such as phase unwrapping for the reconstruction, is time consuming, and thus is often carried out offline. To address this limitation, POPESCU et al. [6] proposed a fast phase reconstruction method (FPRM) by dividing complex matrices with sample and free-sample following Fourier operations, and this can help avoid phase unwrapping when imaging thin samples smaller than $2 \pi$ radians. However, this method still requires spectrum shifting to relieve the car- 
rier, which limits the speedup. To improve the speed of reconstruction, SHAKED et al. [ 7 ] proposed an improved FPRM by employing critical sampling. However, its accuracy thus also decreased due to the sampling. BAI et al. [ $\underline{8}$ ] developed a modified FPRM by encoding the holograms of a sample and free-sample into a synthetic hologram that avoids spectrum shifting. However, limited by the pixel size and number of cameras, the spectrum of the conjugate orders can cause spectrum aliasing with that of the zero order, which reduces the quality of the reconstructed image of the FPRM. Aliasing can be avoided by using a large carrier frequency but this reduces the space-bandwidth product. To eliminate the zero-order term and improve the space-bandwidth product (SBP), slightly off-axis geometries [ $\underline{9}-11]$ were employed. However, due to the need for phase shifting, the setup becomes more complex as a consequence, and either its rate of acquisition or utilization of the field-of-view of the camera decreases. Several digital approaches have been proposed to suppress the zero-order term [12-17], such as nonlinear filtering and the iterative approach. However, most such approaches are time consuming.

In this study, we developed a new FPRM (nFPRM) by combining a simple and fast zero-order term suppression method, the average gray. Through the average gray we could suppress the zero-order term and retrieved the unaltered SBP in Ref. [6] to some extent. Moreover, we need not operate spectrum shifting. We also performed experiments to verify the efficiency of our proposed approach.

\section{Phase reconstruction}

In off-axis $\mathrm{DH}$, the intensity distribution of the hologram acquired by the camera can be expressed as

$$
\begin{aligned}
I(x, y)=d(x, y) & +0.5 m(x, y) \exp \left\{i\left[2 \pi f_{x} x+2 \pi f_{y} y+\varphi(x, y)+\varphi_{\mathrm{b}}(x, y)\right]\right\} \\
& +0.5 m(x, y) \exp \left\{-i\left[2 \pi f_{x} x+2 \pi f_{y} y+\varphi(x, y)+\varphi_{\mathrm{b}}(x, y)\right]\right\}
\end{aligned}
$$

where $d(x, y)$ and $m(x, y)$ denote the zero-order term and the modulated term of the hologram, respectively. They can be regarded as the invariable parameters during the measurement of a pure phase sample, $f_{x}$ and $f_{y}$ denote the carrier frequencies in the $x$ and $y$ directions, respectively, $\varphi(x, y)$ denotes the phase of the measured sample, and $\varphi_{\mathrm{b}}(x, y)$ denotes the unexpected phase introduced by aberration and noise in the system.

Because the hologram is a gray image, its zero-order term $d(x, y)$ can be then approximated to the average gray level of the image in the spatial domain, which has no effect on the measured phase. The zero-order term can thus be filtered out using the grayscale average method for images $[18,19]$ that the image is first divided into many local regions and then the average gray value is calculated for each local region, and the result can be expressed as 


$$
\begin{aligned}
I_{\mathrm{f}}(x, y) & =0.5 m(x, y) \exp \left\{i\left[2 \pi f_{x} x+2 \pi f_{y} y+\varphi(x, y)+\varphi_{\mathrm{b}}(x, y)\right]\right\} \\
& +0.5 m(x, y) \exp \left\{-i\left[2 \pi f_{x} x+2 \pi f_{y} y+\varphi(x, y)+\varphi_{\mathrm{b}}(x, y)\right]\right\}
\end{aligned}
$$

Having applied the Fourier transform (FT) operation to Eq. (2), we can divide the conjugate-orders terms in the spectral domain and choose the expected first-order term through a band-pass filter (BPF). In Ref. []], the first-order term needs to be shifted back to the center to eliminate carrier frequencies, but this operation is not necessary. After filtering, we can directly operate the inverse Fourier transform (IFT) and obtain the result as

$$
\begin{aligned}
r(x, y) & =\operatorname{IFT}\left\{\mathrm{FT}\left\{I_{\mathrm{f}}\right\} \cdot \mathrm{BPF}\right\} \\
& =0.5 m(x, y) \exp \left\{i\left[2 \pi f_{x} x+2 \pi f_{y} y+\varphi(x, y)+\varphi_{\mathrm{b}}(x, y)\right]\right\}
\end{aligned}
$$

When there is no phase sample in the input plane, we repeat the above-mentioned processes and obtain

$$
r_{\mathrm{n}}(x, y)=0.5 m(x, y) \exp \left\{i\left[2 \pi f_{x} x+2 \pi f_{y} y+\varphi_{\mathrm{b}}(x, y)\right]\right\}
$$

where $\varphi_{\mathrm{b}}$ includes the background phase and phase aberration. Note that we can remove $m, \varphi_{\mathrm{b}}, f_{x}$ and $f_{y}$ through a division between Eqs. (3) and (4) to yield a complex function containing only sample information. By performing an arc tangent operation, we can obtain the thin sample phase distribution with the term

$$
\varphi(x, y)=\operatorname{atan} \frac{r(x, y)}{r_{\mathrm{n}}(x, y)}
$$

Similar to Ref. []], our approach can implement thin phase reconstruction with only one arc tangent operation. But our approach abandons spectrum shifting and, in particular, suppresses the zero-order term to yield in an improvement in the SBP [11]. Compared with Refs. [12-16], the suppression does not occur in the spectral domain but in the spatial domain, which can also lead to a higher utilization of the expected first-order term.

\section{Experimental results}

To demonstrate the feasibility of nFPRM, we first imaged a phase plate by using off-axis DH microscopy using a reflective point diffraction interferometer [20]. In the experiments, we used an He-Ne laser with $\lambda=632.8 \mathrm{~nm}$ as a light source and a CCD camera 

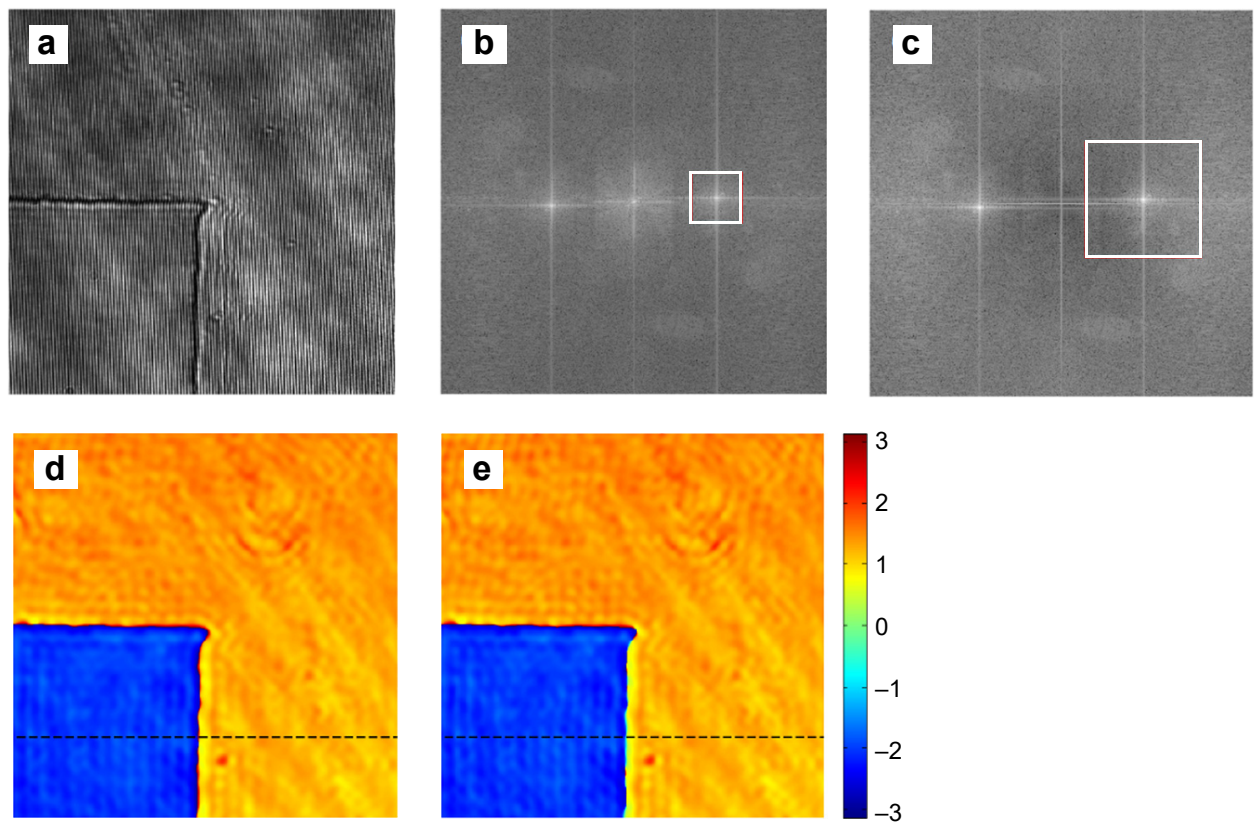

Fig. 1. Experimental results on a phase plate. Hologram (a). Spatial power spectrum obtained by FPRM (b) and nFPRM (c). Phases reconstructed by FPRM (d) and nFPRM (e); the color bar representing units in radians.

with a resolution of $1600 \times 1200$ pixels as a recorder, where each pixel had an area of $4.4 \times 4.4 \mu \mathrm{m}$. Figure 1 a shows the phase plate hologram acquired. The refractive index of the phase plate was 1.5168 and its step height was $580.22 \mathrm{~nm}$ as measured by a BRUKER atomic force microscope. This provided an optical path difference of $2.98 \mathrm{rad}$ at $\lambda=632.8 \mathrm{~nm}$. Using the Fourier transform, we obtained its spatial power spectrum as shown in Fig. $1 \mathbf{b}$. The zero-order term clearly overlapped with the conjugate-order terms in the figure, which caused aliasing. By applying the grayscale aver-

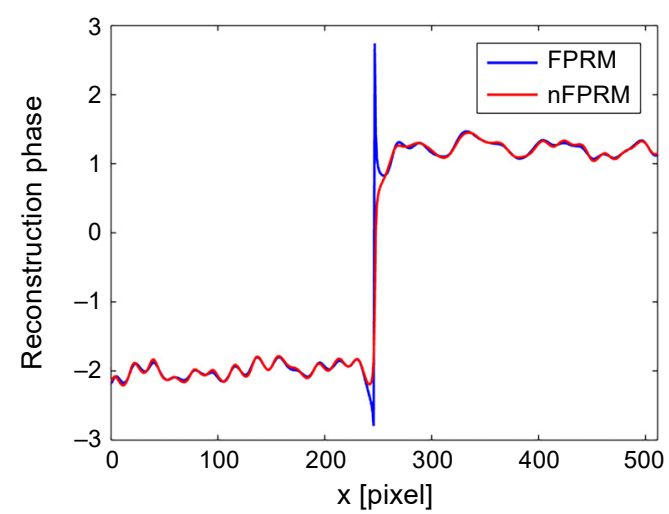

Fig. 2. Comparison profiles along the dashed lines indicated in Figs. 1d and 1e. 
age method to the image, we obtained its new spatial power spectrum as shown in Fig. 1c. Note that we suppressed the zero-order term even though there was a small amount of residual zero-order information in the center, and then enlarged the chosen coverage of the first order. Figure 1d shows the result reconstructed by the FPRM, Fig. 1e illustrates the result obtained by the nFPRM, and Fig. 2 shows the comparison profiles along the dashed lines indicated in Figs. $1 \mathbf{d}$ and $1 \mathrm{e}$. These results revealed that the FPRM suffered from aliasing artifacts while the nFPRM was free of them, especially at the edge of the step. We also carried out a comparison in MATLAB between FPRM and nFPRM using the hologram shown in Fig. 1 a with $512 \times 512$ pixels. The results illustrated that FPRM operated at $30 \mathrm{~ms}$ while the nFPRM operated at $39.9 \mathrm{~ms}$. Our approach also achieved real-time imaging. Like the FPRM, our approach only required a highly parallelizable operation such as the Fourier transform and matrix multiplication. Therefore, a much higher retrieval speed could be allowed by using a GPU.

The quality of the retrieved image is confined by the size of the digital filter (DF) employed in the spectral domain. The maximum allowable size of the DF is determined by the distance between the first- and zero-order terms. Without loss of generality, we define $F_{\mathrm{D}}=1$ as half the length of the square side as shown in Fig. 1c that touches the zero-frequency point [11]. Figure 3 shows the comparison between the performance of FPRM and nFPRM at different values $(0.3,0.5$, and 0.7$)$ of $F_{\mathrm{D}}$. We see that the nFPRM allowed us to use a DF with a much larger $F_{\mathrm{D}}$, which resulted in improved retrieval performance.
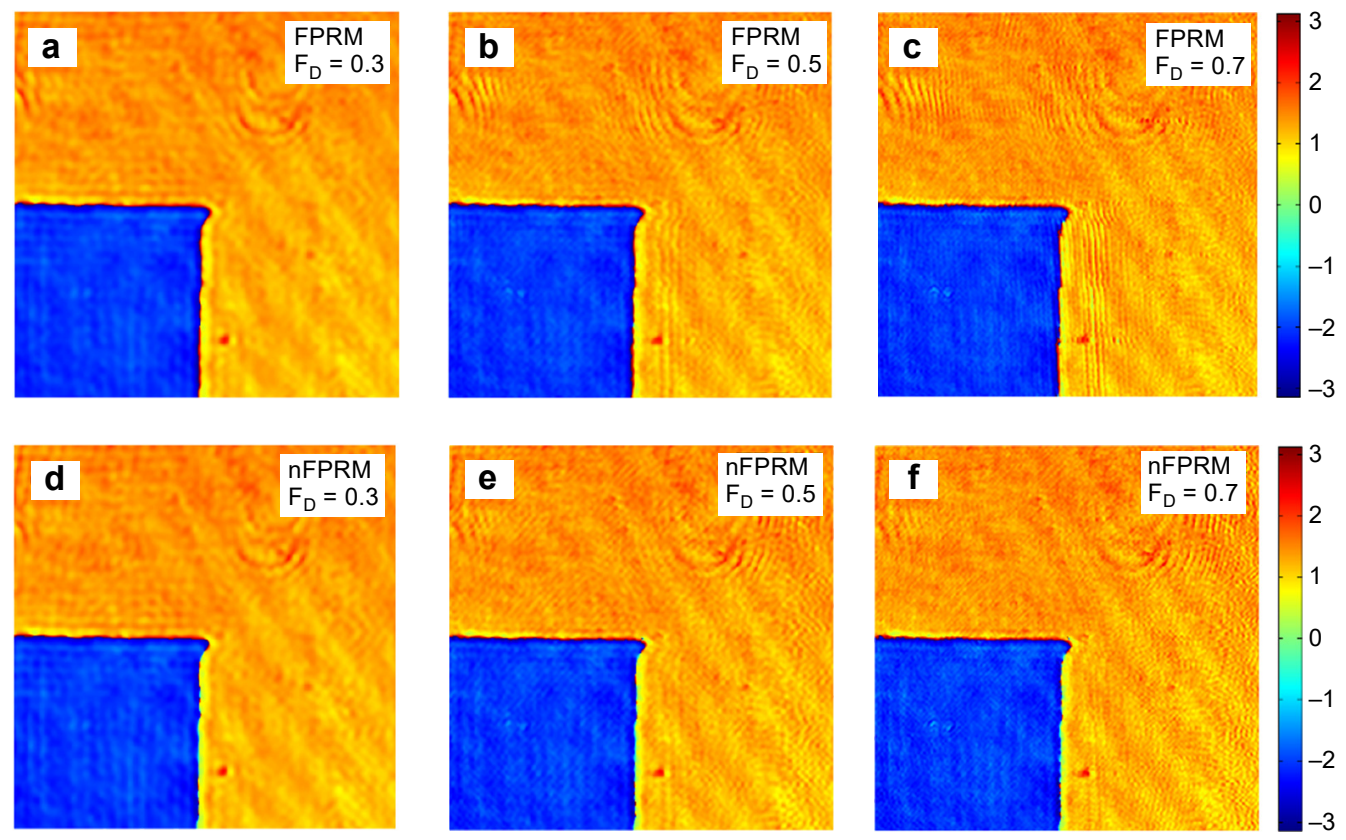

Fig. 3. Phase distributions of a phase plate obtained by FPRM and nFPRM, with the color bar representing units in radians. 

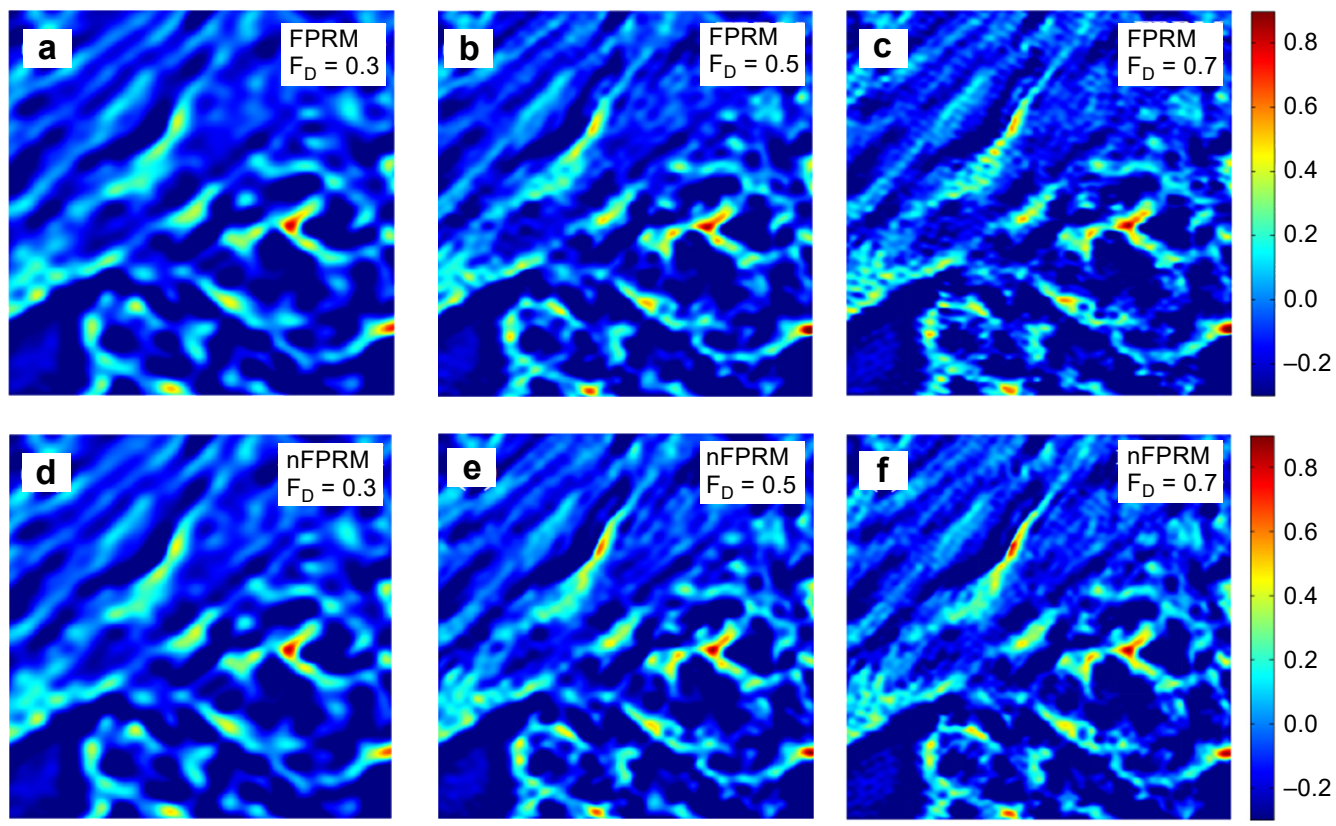

Fig. 4. Phase distributions of an unlabeled prostate tissue obtained by FPRM and nFPRM, with the color bar representing units in radians.

To further verify the effectiveness of the nFPRM, we imaged an unlabeled prostate tissue by using white-light diffraction phase microscopy (wDPM). In wDPM $[11,21,22]$, we employed a halogen lamp commonly used in commercial microscopes as spatially coherent white light illumination and a CCD camera (Hamamatsu ORCA Flash) as a recorder. In order to ensure the field to be spatially coherent over the entire field of view, we need to close the condenser aperture to the value $(\mathrm{NA}=0.09)$. The diffraction grating used in the wDPM was at a period of 33.33 lines $/ \mathrm{mm}$. Figure 4 shows the comparison between FPRM and nFPRM. Again, the nFPRM yielded higher retrieval capability, and the aliasing artifact was noticeable for the FPRM as a result of frequency overlap in the spectral domain.

\section{Conclusion}

In this paper, we proposed and verified an nFPRM for off-axis DH by combining the average gray. The zero-order term was suppressed by the average gray, and the phase was reconstructed using a free-sample hologram. Consequently, the nFPRM quickly reconstructed the phase without spectrum shifting. Moreover, the nFPRM yielded nearly the same speed as the FPRM with better reconstruction capability. Experimental results on an off-axis DH using laser and white light illumination showed the perfor- 
mance of our approach. Higher speeds could be achieved by performing all operations on a GPU. We think our approach is practical for real-time off-axis DH.

Acknowledgments - This work was supported by the Education Department Foundation of Heilongjiang Province of China (grant number 12541584) and Natural Science Foundation of Heilongjiang Province of China (grant number C2018050).

\section{References}

[1] Shan M.G., Liu L., Zhong Z., Liu B., Luan G.Y., Zhang Y.B., Single-shot dual-wavelength off-axis quasi-common-path digital holography using polarization-multiplexing, Optics Express 25(21), 2017, pp. 26253-26261, DOI: 10.1364/OE.25.026253.

[2] Dardikman G., Turko N.A., Nativ N., Mirsky S.K., Shaked N.T., Optimal spatial bandwidth capacity in multiplexed off-axis holography for rapid quantitative phase reconstruction and visualization, Optics Express 25(26), 2017, pp. 33400-33415, DOI: 10.1364/OE.25.033400.

[3] GuO R.L., WANG F., Hu X.Y., YANG W.Q., Off-axis low coherence digital holographic interferometry for quantitative phase imaging with an LED, Journal of Optics 19(11), 2017, article 115702, DOI: $10.1088 / 2040-8986 /$ aa8887.

[4] Guo C.S., Wang B.Y., Sha B., Lu Y.J., Xu M.Y., Phase derivative method for reconstruction of slightly off-axis digital holograms, Optics Express 22(25), 2014, pp. 30553-30558, DOI: 10.1364 / OE.22.030553.

[5] Wang M.Q., Wang K., Zheng M., Li F., Wu J., Dual reference beam digital off-axis holography for exact suppression of zero-order term and twin image without spectrum loss, Optica Applicata 47(3), 2017, pp. 435-443, DOI: 10.5277/oa170309.

[6] Pham H.V., Edwards C., Goddard L.L., Popescu G., Fast phase reconstruction in white light diffraction phase microscopy, Applied Optics 52(1), 2013, pp. A97-A101, DOI: 10.1364/AO.52.000A97.

[7] Girshovitz P., SHAKed N.T., Real-time quantitative phase reconstruction in off-axis digital holography using multiplexing, Optics Letters 39(8), 2014, pp. 2262-2265, DOI: 10.1364/OL.39.002262.

[8] Bai H.Y., Shan M.G., Zhong Z., Guo L.L., Zhang Y.B., Liu B., Fast and accurate carrier and aberration removal in phase retrieval for off-axis holography, Optik 162, 2018, pp. 95-101, DOI: 10.1016/j.ijleo.2018.02.070.

[9] Zhong Z., Bai H.Y., Shan M.G., Zhang Y.B., Guo L.L., Fast phase retrieval in slightly off-axis digital holography, Optics and Lasers in Engineering 97, 2017, pp. 9-18, DOI: 10.1016/j.optlaseng. 2017.05.004.

[10] Hao B.G., Shan M.G., Zhi Z., Diao M., Wang Y., Zhang Y.B., Parallel two-step spatial carrier phase-shifting interferometric phase microscopy with fast phase retrieval, Journal of Optics 17(3), 2015, article 035602, DOI: 10.1088/2040-8978/17/3/035602.

[11] Shan M.G., Kandel M.E., Majeed H., Nastasa V., Popescu G., White-light diffraction phase microscopy at doubled space-bandwidth product, Optics Express 24(25), 2016, pp. 29033-29039, DOI: 10.1364/OE.24.029033.

[12] Pavillon N., Seelamantula C.S., Kuhn J., Unser M., Depeursinge C., Suppression of the zero-order term in off-axis digital holography through nonlinear filtering, Applied Optics 48(34), 2009, pp. H186-H195, DOI: 10.1364/AO.48.00H186.

[13] Li J.C., Peng Z.J., Tankam P., Song Q.H., Picart P., Digital holographic reconstruction of a local object field using an adjustable magnification, Journal of the Optical Society of America A 28(6), 2011, pp. 1291-1296, DOI: 10.1364/JOSAA.28.001291.

[14] Pavillon N., Arfire C., Bergoend I., Depeursinge C., Iterative method for zero-order suppression in off-axis digital holography, Optics Express 18(15), 2010, pp. 15318-15331, DOI: 10.1364 / OE.18.015318. 
[15] Ma Z.H., Deng L.J., Yang Y., Zhai H.C., Ge Q., Numerical iterative approach for zero-order term elimination in off-axis digital holography, Optics Express 21(23), 2013, pp. 28314-28324, DOI: 10.1364/OE.21.028314.

[16] Zhao D.L., XIE D.Z., YANG Y., Zhai H.C., Iterative approach for zero-order term elimination in off-axis multiplex digital holography, Optics Communications 383, 2017, pp. 513-517, DOI: 10.1016/j.optcom.2016.09.049.

[17] Lu W.L., ChAng M., Chen P.C., Luo W.M., Iterative deconvolution technique for measurements of diffraction-limited images on optical microscopes, Journal of Modern Optics 61, 2014, pp. S2-S9, DOI: $10.1080 / 09500340.2014 .924596$.

[18] Sahoo S.K., Suresh N., VARMA A.K., Correlation between average gray value (AGV) of coal froth image with its ash and vitrinite content, Energy Sources, Part A: Recovery, Utilization, and Environmental Effects 39(9), 2017, pp. 893-898, DOI: 10.1080/15567036.2016.1273280.

[19] Yi J.Z., Mao X., Chen L.J., Xue Y.L., Compare A., Illuminant direction estimation for a single image based on local region complexity analysis and average gray value, Applied Optics 53(2), 2014, pp. 226-236, DOI: 10.1364/AO.53.000226.

[20] Bai H.Y., Zhong Z., Shan M.G., Liu L., Guo L.L., Zhang Y.B., Interferometric phase microscopy using slightly-off-axis reflective point diffraction interferometer, Optics and Lasers in Engineering 90, 2017, pp. 155-160, DOI: 10.1016/j.optlaseng.2016.10.011.

[21] Bhaduri B., Pham H., Mir M., Popescu G., Diffraction phase microscopy with white light, Optics Letters 37(6), 2012, pp. 1094-1096, DOI: 10.1364/OL.37.001094.

[22] Рham H., Bhaduri B., Ding H.F., Popescu G., Spectroscopic diffraction phase microscopy, Optics Letters 37(16), 2012, pp. 3438-3440, DOI: 10.1364/OL.37.003438.

Received June 10, 2019

in revised form August 27, 2019 